\title{
Two-dimensional dynamics of expansion of a degenerate Bose gas
}

\author{
Igor E. Mazets ${ }^{1,2}$ \\ ${ }^{1}$ Vienna Center for Quantum Science and Technology, Atominstitut, TU Wien, 1020 Vienna, Austria \\ ${ }^{2}$ Ioffe Physico-Technical Institute of the Russian Academy of Sciences, 194021 St.Peterburg, Russia
}

\begin{abstract}
Expansion of a degenerate Bose gas released from a pancake-like trap is numerically simulated under assumption of separation of motion in the plane of the loose initial trapping and the motion in the direction of the initial tight trapping. The initial conditions for the phase fluctuations are generated using the extension to the two-dimensional case of the description of the phase noise by the Ornstein-Uhlenbeck stochastic process. The numerical simulations, taking into account both the finite size of the two-dimensional system and the atomic interactions, which cannot be neglected on the early stage of expansion, did not reproduce the scaling law for the peaks in the density fluctuation spectra experimentally observed by Choi, Seo, Kwon, and Shin [Phys. Rev. Lett. 109, 125301 (2012)]. The latter experimental results may thus require an explanation beyond our current assumptions.
\end{abstract}

PACS numbers: 67.85.-d,03.75.Lm,03.75.Hh

Correlations in degenerate Bose gases with repulsive interactions manifest themselves most apparently via phase coherence. Even low-dimensional degenerate bosonic systems (quasicondensates) demonstrate phase coherence over finite distances. After release from the trap, phase fluctuations are converted, in the course of free expansion, into density-density correlations (density ripples). Theory for expansion of both one-dimensional (1D) and two-dimensional (2D) trapping geometries has been developed [1] and experimentally proven for 1D trapped gases [2] (similar behavior has been previously found in expanding clouds, which were in the threedimensional Bose-Einstein condensate rather in the 1D regime inside a very elongated trap [3] ).

In contrast to 1D Bose gases, where correlations decay exponentially and their characteristic length $\lambda_{T}$ determines the typical wavelength scale of the density ripples and the timescale $m \lambda_{T}^{2} / \hbar$ of their emergence ( $m$ being the mass of the atom), correlations in degenerate 2D Bose gases decay with the distance according to a power law, if the temperature is below the point of the BeresinskiiKosterlitz-Thouless (BKT) transition [4]. Such a powerlaw decay renders no specific correlation length, the spectrum of the density fluctuations of a $2 \mathrm{D}$ gas released from a trap evolves in a self-similar way at asymptotically large expansion times $t_{\mathrm{e}}$. In particular, after averaging the power spectrum of density fluctuations

$$
P_{\mathbf{q}}=\left|\int d^{2} \boldsymbol{\rho} \delta n(\boldsymbol{\rho}) e^{-i \mathbf{q} \rho}\right|^{2}
$$

where $\delta n(\boldsymbol{\rho})$ is the local density fluctuation, over the directions of the $2 \mathrm{D}$ wave vector $\mathbf{q}$, the $n$th peak position $q_{n}, n=1,2,3, \ldots$, is expected to satisfy the relation [1]

$$
\hbar q_{n}^{2} t_{\mathrm{e}} /(2 \pi m)=n-1 / 2 .
$$

Recently, Choi et al. [5] reported their experimental results on probing fluctuations in a $2 \mathrm{D}$ gas of bosonic $\left({ }^{23} \mathrm{Na}\right)$ atoms in free expansion after a sudden release from a pancake-like optical trap, the trapping frequencies being equal to $\left(\omega_{x}, \omega_{y}, \omega_{z}\right)=2 \pi \times(3.0,3.9,370) \mathrm{Hz}$. The chemical potential was about $\mu_{0}=2 \pi \hbar \times 260 \mathrm{~Hz}$, i.e., less than the spacing between the discrete levels of the trapping Hamiltonian in the tighgtly confined direction. The coherent fraction of atomic ensemble decreased from 0.78 to 0.12 as the temperaure $T$ grew from $20 \mathrm{nK}$ up to the BKT transition temperature $T_{\mathrm{BKT}} \approx 70 \mathrm{nK}$.

The experimental results [5] turned out to be quite surprising. Instead of recovering the scaling (2), they brought about another dependence,

$$
\hbar q_{n}^{2} t_{\mathrm{e}} /(2 \pi m)=\alpha n^{\gamma},
$$

with $0.2<\alpha<0.45$ and $0.7<\gamma<1$ for expansion times in the range $10 \mathrm{~ms}<t_{\mathrm{e}}<25 \mathrm{~ms}$. In other words, the spectral peaks of the density fluctuations were not only shifted with respect to Eq. (2), but the spacing between the ajacent peaks was significantly smaller than Eq. (2) predicts. Choi et al. ruled out the effects of the cloud finite size and suggested that such an unexpected behavior might be intrinsic to the expansion dynamics [5].

To check this assumption, we performed numerical simulations of 2D expansion of a degenerate gas. Since the temperature and, hence, the coherent fraction do not affect significantly the peak positions $q_{n}$ [5], we find it safe to neglect thermal population of the excited levels of motion in the tightly trapped $(z)$ direction and model the system's dynamics by the Gross-Pitaevskii equation (GPE), taking into account the interaction effects, which are important at the initial stage of expansion. Furthemore, we separate the motion in $z$-direction and in the $(x, y)$-plane. This would be impossible if the Beliaev and Landau damping of elementary excitations in expanding but still dense enough atomic cloud were giving rise to significant population of modes with nonzero $z$-components of kinetic momentum. However, the estimation of the ratio of the damping rate of an elementary excitation to its frequency yields [6] the value $\sim a_{s}^{1 / 2} /\left(n_{3 \mathrm{D}}^{1 / 2} \lambda_{\mathrm{th}}^{2}\right) \sim 10^{-2}$, where $a_{s}$ is the atomic $s$-wave scattering length, $n_{3 \mathrm{D}}$ is the three-dimensional atomic number density, and $\lambda_{\mathrm{th}}$ is the de Broglie wave length of 
atoms at temperature $T$. Even for elementary excitations with the energy close to the chemical potential the typical relaxation time (and, hence, the characteristic time of scattering into $z$-direction) in a three-dimensional degenerate gas with the bulk density equal to the peak density in our 2D system is of the order of $100 \mathrm{~ms}$, which is much longer than the typical expansion time. Therefore we can separate the motion in different directions. In $z$ direction, we have a free expansion of a wave packet, which is initially, at $t=0$, the wave function of the ground state of the harmonic trapping potential with the fundamental frequency $\omega_{z}$. Then the motion in the $(x, y)$ plane is described by the GPE

$$
i \hbar \frac{\partial}{\partial t} \Psi=-\frac{\hbar^{2}}{2 m}\left(\frac{\partial^{2}}{\partial x^{2}}+\frac{\partial^{2}}{\partial y^{2}}\right) \Psi+\mu(t)|\Psi|^{2} \Psi .
$$

The fast (but still not infinitely fast) evolution of the quasicondensate profile in $z$-direction affects the slow motion in the perpendicular plane) only through the time-dependent nonlinearity in Eq. (4). The timedependent effective $2 \mathrm{D}$ coupling constant $\mu(t)$ takes into account the growth of the transverse density profile width $\propto \sqrt{1+\left(\omega_{z} t\right)^{2}}$. The normalization condition $\max |\Psi(x, y, t=0)|=|\Psi(x=0, y=0, t=0)| \equiv 1$ fixes the prefactor, and we obtain

$$
\mu(t)=\frac{\mu_{0}}{\sqrt{1+\left(\omega_{z} t\right)^{2}}} .
$$

Initial shape of $|\Psi|^{2}$ is the inverted-parabolic ThomasFermi profile corresponding to the chemical potential $\mu_{0}$ of the trapped gas of sodium atoms. The phase $\varphi=$ $\arg \Psi$ at $t=0$ can be generated using the generalization of the stochastic Ornstein-Uhlenbeck process, previously used to model random phase distributions of phases in 1D quasicondensates [7, 8].

Assume that the temperature $T$ of $2 \mathrm{D}$ Bose gas is below the BKT transition, phase and density fluctuations are small and, therefore, the Hamiltonian can be linearized:

$$
H=\frac{\hbar^{2}}{2 m} \int d^{2} \boldsymbol{\rho}\left[n_{2 \mathrm{D}}(\boldsymbol{\rho})\left(\nabla_{\perp} \varphi\right)^{2}+\tilde{g} \delta n^{2}+\frac{\left(\nabla_{\perp} \delta n\right)^{2}}{4 n_{2 \mathrm{D}}(\boldsymbol{\rho})}\right]
$$

Here $n_{2 \mathrm{D}}=\int d z n_{3 \mathrm{D}}$ is the $2 \mathrm{D}$ number density, $\tilde{g}$ is the dimensionless coupling strength $(\tilde{g} \approx 0.013$ in the experiment [5] $), \nabla_{\perp}$ is the 2D gradient in the $(x, y)$-plane. Then the partition function of a trapped gas is

$$
Z=\int \mathcal{D} \varphi \int \mathcal{D} \delta n \exp \left[-H /\left(k_{\mathrm{B}} T\right)\right]
$$

The density fluctuation variable can be integrated out, and we obtain

$$
Z=\mathrm{const} \int \mathcal{D} \varphi \exp \left[-\frac{\hbar^{2}}{2 m k_{\mathrm{B}} T} \int d^{2} \boldsymbol{\rho} n_{2 \mathrm{D}}(\boldsymbol{\rho})\left(\nabla_{\perp} \varphi\right)^{2}\right] .
$$

We can use the thermodynamic expression (8) to generate initial phase distribution for individual realizations of our numerical simulations. On a $(2 M+1) \times(2 M+1)$ square grid Eq. (8) takes the form

$$
\begin{aligned}
Z= & \text { const } \prod_{l_{x}=-M}^{M} \prod_{l_{y}=-M}^{M} \int d \varphi_{l_{x} l_{y}} \exp \left\{-\frac{\varepsilon}{2} w_{l_{x} l_{y}} \times\right. \\
& {\left.\left[\left(\varphi_{l_{x} l_{y}}-\varphi_{l_{x} l_{y}-1}\right)^{2}+\left(\varphi_{l_{x} l_{y}}-\varphi_{l_{x}-1 l_{y}}\right)^{2}\right]\right\} . }
\end{aligned}
$$

Here the pair of subscripts $l_{x}$ and $l_{y}$ denotes a variable at the point with coordinates $x=l_{x} \Delta s$ and $y=l_{y} \Delta s$ [note that the grid step $\Delta s$ does not appear in Eq. (9) in our 2D problem]. The density profile and its peak value are characterized by dimensionless quantities $w_{l_{x} l_{y}}=n_{2 \mathrm{D}}\left(l_{x} \Delta s, l_{y} \Delta s\right) / n_{2 \mathrm{D}}(0,0)$ and $\varepsilon=\hbar^{2} n_{2 \mathrm{D}}(0,0) /\left(m k_{\mathrm{B}} T\right)$, respectively. Periodic boundary conditions are set, i.e., $\varphi_{l_{x} M+1}=\varphi_{l_{x}-M}$ and $\varphi_{M+1 l_{y}}=\varphi-M l_{y}$.

Assume we know the $2 M+1$ phases $\varphi_{l_{x}-1 l_{y}}$. Then we can calculate the phases $\varphi_{l_{x} l_{y}}$ in the next row of the grid using the transfer matrix approach [9].

We assume that the grid step is small enough and thus we can write $w_{l_{x} l_{y}} / w_{l_{x} 0} \approx w_{l_{x}-1 l_{y}} / w_{l_{x}-10}$. The transformation [10]

$$
A_{l_{x}-1 j}=\sum_{l_{y}=-M}^{M} V_{l_{x} ; j l_{y}} \sqrt{\frac{w_{l_{x} l_{y}}}{w_{l_{x} 0}}} \varphi_{l_{x}-1 l_{y}}
$$

diagonalizes the non-negative quadratic form

$$
\sum_{l_{y}=-M}^{M} \frac{w_{l_{x} l_{y}}}{w_{l_{x} 0}}\left(\varphi_{l_{x}-1 l_{y}}-\varphi_{l_{x}-1 l_{y}-1}\right)^{2}=\sum_{j=0}^{2 M} J_{l_{x} j} A_{l_{x}-1 j}^{2} .
$$

Both $V_{l_{x} ; j l_{y}}$ and $J_{l_{x} j}$ can found numerically by standard methods of linear algebra. Then Eq. (9) factorizes: $Z=$ const $\prod_{j=0}^{2 M} Z_{j}$, where each factor

$$
\begin{aligned}
Z_{j}= & \prod_{l_{x}=-M}^{M} \int d A_{l_{x} j} \exp \left[-\frac{\varepsilon_{l_{x}}}{2}\left(A_{l_{x}-1 j}-A_{l_{x} j}\right)^{2}-\right. \\
& \left.\frac{\varepsilon_{l_{x}} J_{l_{x} j}}{2} A_{l_{x} j}^{2}\right]
\end{aligned}
$$

corresponds to a $1 \mathrm{D}$ evolution (along the $x$-coordinate) of a $A_{j}$ describable by the Ornstein-Uhlenbeck stochastic process (which can be easily seen from the analogy with the relative phase between two tunnel-coupled 1D quasicondensates at thermal equilibrium [7]). Here $\varepsilon_{l_{x}}=\varepsilon w_{l_{x} 0}$. The formula [1]

$$
A_{l_{x} j}=A_{l_{x}-1 j} e^{-\sqrt{J_{l_{x} j}}}+\sqrt{\frac{1-e^{-2 \sqrt{J_{l_{x} j}}}}{2 \varepsilon_{l_{x}} \sqrt{J_{l_{x} j}}}},
$$

where $\varsigma$ is a (pseudo)random number obeying the Gaussian distribution with zero mean and unity variance, updates the variable $A_{j}$ on the next step. Then we obtain 


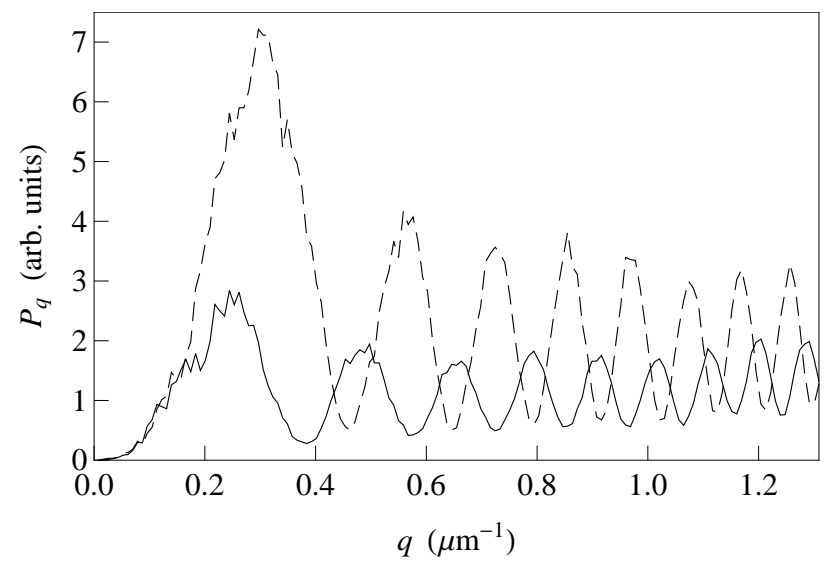

FIG. 1: Solid line: the power spectrum of density fluctuations for $t_{\mathrm{e}}=11 \mathrm{~ms}$ obtained from Eq. (4), i.e., taking into account interaction of atoms in an expanding atomic cloud. Parameters of the trapped degenerate cloud are taken from Ref. [5] (see details in the text). Dashed line: spectrum of density fluctuations obtained for a purely ballistic expansion during the same $t_{\mathrm{e}}$. The spectra are averaged over 5 realisations each.

the initial phase values in the next grid row:

$$
\varphi_{l_{x} l_{y}}=\sum_{l_{y}=-M}^{M} V_{l_{x} ; j l_{y}} \sqrt{\frac{w_{l_{x} 0}}{w_{l_{x} l_{y}}}} A_{l_{x} j} .
$$

Subsequent steps are done using the new local values for the density profile. The same procedure can be used to obtain $A_{l_{x}-2 j}$ from the known $A_{l_{x}-1 j}$.

Eq. (13), together with chosing the (pseudo)random initial values of the $A_{l_{x}} j$ variables for a certain row (e.g., $\left.l_{x}=0\right)$ according to a Gaussian distribution with zero mean and variance $\left\langle A_{l_{x} j}^{2}\right\rangle=1 /\left(2 \varepsilon_{l_{x}} \sqrt{J_{l_{x} j}}\right)$, enables us to prepare the initial conditions for each realization of our numerical modeling [12].

We solved Eq. (4) using the split-step pseudospectral method for the typical parameters of Ref. [5] and obtained the power spectra of density fluctuations. As the density fluctuation $\delta n$ in Eq. (1) we took the difference between the local densities given at the same expansion time $t_{\mathrm{e}}$ by two solutions, one containing initial phase fluctuations and other having constant phase everywhere at $t=0$. An example of such a spectrum $P_{q}$, that is $P_{\mathbf{q}}$ averaged over the direction of the wave vector $\mathbf{q}$, is displayed in Fig. 1. The variation of the temperature from $60 \mathrm{nK}$ to $20 \mathrm{nK}$ (corresponding to the increase of $\varepsilon$ from 15 to 45) changes the height of the peaks of $P_{q}$ only, leaving the peak positions $q_{n}$ unmodified, as we can expect from the experimental results [5]. The solution of the GPE (4) is also juxtaposed with the density fluctuations spectra for purely ballistic expansion [i.e., by setting $\mu(t) \equiv 0$ ]. In the case of purely ballistic expansion, we found that Eq. (2) is satisfied with $1 \%$ accuracy for $n \geq 2$; for the first peak position we obtained $\hbar q_{1}^{2} t_{\mathrm{e}} /(2 \pi m) \approx 0.4$ for $10 \mathrm{~ms} \leq t_{\mathrm{e}} \leq 23 \mathrm{~ms}$.

As we can see from Fig. 1 interaction of atoms during expansion is not negligible and shifts the peak positions with respect to the ballistic expansion case. However, instead of recovering Eq. (3), we found that the peak positions are well described by the formula

$$
\hbar q_{n}^{2} t_{\mathrm{e}} /(2 \pi m)=\beta(n-\ell),
$$

where

$$
\beta \approx 0.9, \quad \ell \approx 0.7
$$

for the whole range of experimentally relevant expansion times (see Fig. 22).

Note, that the transversal trapping frequency $\omega_{z}=$ $2 \pi \times 370 \mathrm{~Hz}$ of Ref. [5] is approximately equal to the radial trapping frequency in the experiment of Ref. [3], but the cigar-shaped configuration of the atomic cloud resulted in the latter case in a faster $\left(\propto t^{-2}\right.$ instead of $\propto t^{-1}$ at expansion times $\gtrsim 1 \mathrm{~ms}$ ) decrease of the threedimensional density of atoms than in the $2 \mathrm{D}$ case. Radial trapping frequencies in recent experiments with 1D ultracold gases on atom chips [2, 8] were by an order of magnitude higher, thus further decreasing the time scale, on which atomic interactions still play a role. Therefore expansion of atomic clouds in the experiments [2, 3, 8 ] was very close to ballistic, in contrast to the case modeled in our present work.

Our numerical results do not reproduce the observations by Choi et al. [5]. For example, our calculations predict the values of the fifth peak position $q_{5}$, which are at relevant expansion times larger by a factor 2-3 than the observed ones. In general, our calculations predict the linear depenfdence of $q_{n}^{2}$ on the peak number $n$, in contrast to the nonlinear fitting formula (3) found in Ref. [5].

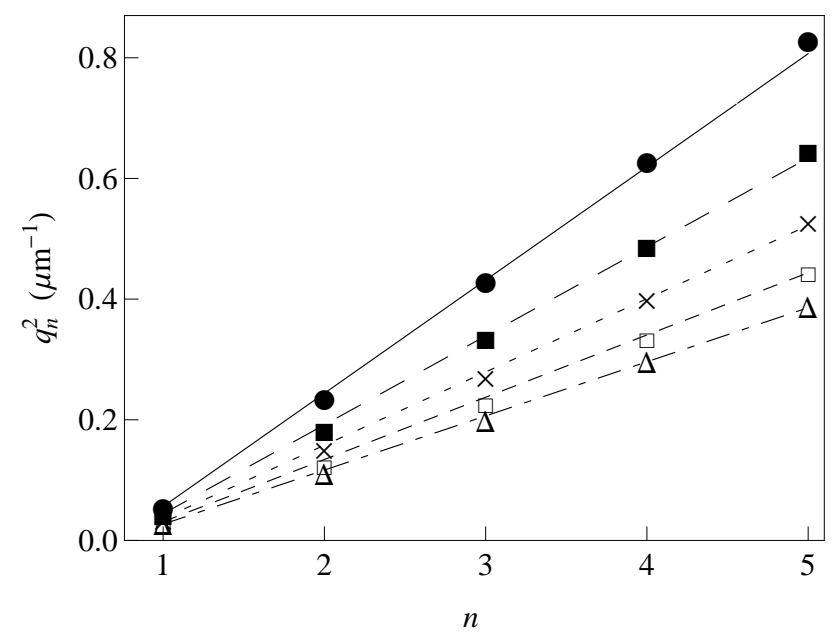

FIG. 2: The peak positions for the power spectrum of density fluctuations (the peak number $n$ is dimensionless) of a ${ }^{23} \mathrm{Na}$ ultracold gas interacting during the expansion for $t_{\mathrm{e}}=11 \mathrm{~ms}$ (filled circles), $14 \mathrm{~ms}$ (filled squares), $17 \mathrm{~ms}$ (crosses), $20 \mathrm{~ms}$ (open squares), and $23 \mathrm{~ms}$ (triangles). Straight lines (solid, long-dashed, dotted, short-dashed, and dot-dashed, respectively) display the fitting by Eqs. (15, 16). 
Such a conspicuous disagreement between the theory that assumes separation of motion in the former trap plane and in the direction of the tight confinement before the release of the atom from the trap and the experimental results [5] calls for explanantion. We do not expect the scattering of phonons into $z$-direction in the expanding quasicondensate to be a reason for such a discrepancy, since the estimations based on the theory of Ref. [6] show that this process is too slow compared to the typical expansion times. Moreover, the influence of such a scattering would grow with $t_{\mathrm{e}}$, but our results de- viate strongly from the experimental findins at shorter times as strongly as at longer ones.

The fact that the experimental spectra of density fluctuations are describable by Eq. (3) when no vortices are present in the $2 \mathrm{D}$ system [5] allow us to rule out any vortex-related explanation of this discrepancy, which therefore still remains puzzling.

This work was supported by the FWF (project P 22590-N16). The author thanks J. Schmiedmayer and Yong-il Shin for helpful discussions.
[1] A. Imambekov, I. E. Mazets, D. S. Petrov, V. Gritsev, S. Manz, S. Hofferberth, T. Schumm, E. Demler, and J. Schmiedmayer, Phys. Rev. A 80, 033604 (2009).

[2] S. Manz, R. Bücker, T. Betz, Ch. Koller, S. Hofferberth, I. E. Mazets, A. Imambekov, E. Demler, A. Perrin, J. Schmiedmayer, and T. Schumm, Phys. Rev. A 81, 031610(R) (2010).

[3] S. Dettmer, D. Hellweg, P. Ryytty, J. J. Arlt, W. Ertmer, and K. Sengstock, D. S. Petrov, G. V. Shlyapnikov, H. Kreutzmann, L. Santos, and M. Lewenstein, Phys. Rev. Lett. 87, 160406 (2001).

[4] V. L. Berezinskii, Sov. Phys. JETP 34, 610 (1972); J. M. Kosterlitz and D. J. Thouless, J. Phys. C 61181 (1973).

[5] Jae-yoon Choi, Sang Won Seo, Woo Jin Kwon, and Yongil Shin, Phys. Rev. Lett. 109, 125301 (2012).

[6] L.P. Pitaevskii and S. Stringari, Phys. Lett. A 235, 398 (1997); C. J. Pethick and H. Smith, Bose-Einstein Condensation in Dilute Gases, 2nd edition (Cambridge University Press, Cambridge, 2008), p.313.
[7] H.-P. Stimming, N. J. Mauser, J. Schmiedmayer, and I. E. Mazets, Phys. Rev. Lett. 105, 015301 (2010).

[8] T. Betz, S. Manz, R. Bücker, T. Berrada, Ch. Koller, G. Kazakov, I. E. Mazets, H.-P. Stimming, A. Perrin, T. Schumm, and J. Schmiedmayer, Phys. Rev. Lett. 106, 020407 (2011).

[9] J. Zinn-Justin, Quantum Field Theory and Critical Phenomena, 4th edition (Clarendon, Oxford, 2002), Ch. 2 and 23.

[10] The relations $\sum_{l_{y}=-M}^{M} V_{l_{x} ; j l_{y}} V_{l_{x} ; j^{\prime} l_{y}}=\delta_{j j^{\prime}}$ and $\sum_{j=0}^{2 M} V_{l_{x} ; j l_{y}} V_{l_{x} ; j l_{y}^{\prime}}=\delta_{l_{y} l_{y}^{\prime}}$ hold.

[11] D. T. Gillespie, Phys. Rev. E 54, 2084 (1996).

[12] As a consistency check of our method, we can demonstrate that the description via the Ornstein-Uhlenbeck stochastic process yields the correct correlation function of a uniform $2 \mathrm{D}$ gas $\langle\exp [i \varphi(0,0)-i \varphi(x, y)]\rangle \propto$ $\left(\sqrt{x^{2}+y^{2}}\right)^{-1 /(2 \pi \varepsilon)}$ 